\title{
Osseous Ankle Morphology and Recurrent Lateral Sprains: a Case-Control Study
}

\author{
V. Nabii ${ }^{1}$ M. N. Doral ${ }^{2}$, O. Bilge ${ }^{3}$, G. Huri ${ }^{4}$, F. Familiari ${ }^{5}$, J. Nyland ${ }^{6}$ \\ 1 Ministry Of Health, Department of Orthopaedics and Traumatology, University of Health Science, Antalya \\ Research and Education Hospital, Antalya, Turkey \\ 2 School of Medicine, Department of Orthopaedics and Traumatology, Ufuk University, Dr. Ridvan Ege Hospital, \\ Ankara, Turkey \\ 3 Department of Orthopaedics and Traumatology, Department of Sports Medicine, Meram School of Medicine, \\ N.E. University Konya, Konya, Turkey \\ 4 Department of Orthopaedics and Traumatology, School of Medicine, Hacettepe University, Ankara, Turkey \\ ${ }^{5}$ Department of Orthopaedics and Traumatology, Magna Græcia University of Catanzaro, Catanzaro, Italy \\ 6 Department of Orthopaedic Surgery, University of Louisville and Spalding University, Louisville (KY), U.S.A.
}

\section{CORRESPONDING AUTHOR:}

Mahmut Nedim Doral

Department of Orthopaedics and

Traumatology

School of Medicine, Ufuk University

Dr. Rıdvan Ege Hospital

Mevlana Bulvarı (Konya Yolu) No:86-88

06520 Balgat, Ankara, Turkey

E-mail: mndoral@gmail.com

DOI:

10.32098/mltj.03.2021.06

LEVEL OF EVIDENCE: 3

\section{SUMMARY}

Background. This study evaluated the relationship between intrinsic osseous ankle morphological characteristics and recurrent lateral ankle sprain history.

Methods. Fifty-two patients with recurrent lateral ankle sprain history were age- and sex-matched with 46 healthy control group subjects. Standardized anterior-posterior and lateral ankle-foot radiographs were taken and morphometric tibial arclength (TiAL), ankle stability angle $(\gamma)$, sagittal tibial mortise radius (SRTi), trochlea tali length (TaAL), talar tenon height $(\mathrm{h})$, sagittal radius of the trochlea tali arc (SRTa), tibiotalar sector (a), and talar height $(\mathrm{H})$ measurements were performed by an independent radiologist. Stepwise multiple regression, receiver operator characteristics (ROC) and area under the curve (AOC) analyses helped to determine clinical decision-making efficacy.

Results. SRTi:SRTa and TaAL:TiAL ratios displayed strong efficacy for clinical decision-making. The AOC for the SRTi:SRTa ratio was 0.95 (95\% confidence interval of $0.93-1.0$ ). For the SRTi:SRTa ratio, a value of 0.68 produced a sensitivity of 0.96 and a specificity of 0.85 . The AOC for the TaAL:TiAL ratio was 0.97 (95\% confidence interval of 0.89-1.0). For the TaAL: TiAL ratio, a value of 1.15 produced a sensitivity of 0.98 and a specificity of 0.91 .

Conclusions. SRTi:SRTa and TaAL:TiAL ratio intrinsic osseous ankle morphological characteristics were related to recurrent lateral ankle sprain history providing excellent sensitivity and good specificity. Intrinsic osseous ankle morphological characteristics can help guide doctors and surgeons as they make treatment decisions for patients with recurrent lateral ankle sprain injuries.

\section{KEY WORDS}

Ankle; chronic instability; morphometry; sprain; Talo-crural joint; X-rays. 


\section{INTRODUCTION}

Ankle injuries are one of the most common injuries in sports and daily activities, and $77 \%$ of ankle sprains involve the lateral compartment (1). The ankle joint complex comprises contractile and non-contractile anatomical structures that contribute to its function and stability (2). Although ankle anatomy is well known, intrinsic osseous ankle morphological characteristics that contribute to sprain injury risk have not been as well described (3,4). Several intrinsic (e.g., rearfoot alignment, capsuloligamentous laxity, neuromuscular control) and extrinsic risk factors (e.g., shoe type, sports participation, surface conditions) are known to contribute to chronic ankle instability (5). Although often managed successfully with stabilization surgery or conservative physiotherapy, up to $20-40 \%$ of patients who sustain a lateral ankle sprain experience injury recurrence (6). This concern has prompted many researchers to attempt to improve their understanding for how additional intrinsic osseous ankle morphological characteristics might contribute to ankle instability.

The purpose of this study was to evaluate the relationship between osseous ankle morphological characteristics and recurrent lateral ankle sprain history. The study hypothesis was that specific intrinsic osseous ankle morphological characteristics predict recurrent lateral ankle sprain injury history.

\section{MATERIALS AND METHODS}

The Ethics Committee of the Medical Sciences (IRB) approved the present study and participants provided written informed consent prior to study participation. Between July 2009 and January 2015, 52 (18 men and 34 women) patients with a history of recurrent lateral ankle sprain injuries were recruited from the Hacettepe University, Department of Orthopaedics and Traumatology (table I).

After ankle injury, initial treatment was conservative, such as functional rehabilitation (7-9). Chronic ankle instability, which is characterized by persistent pain, instability, re-injury and permanent functional disability, may develop in $50 \%$ of patients (10). Surgical treatment may be an option in patients with chronic ankle instability who fail appropriate conservative therapy after more than 6 months (11). Inclusion and exclusion criteria was conducted based on the International Ankle Consortium recommendations (10). Only patients who had undergone lateral ankle ligament reconstruction after experiencing $\geq 3$ recurrent lateral ankle sprains were included in the case group. To be included for study participation subjects had to have experienced a "buckling" or "giving way" episode within three months prior to surgery $(10,12)$. Study exclusion criteria included the presence of any new acute lower extremity injury in the last 3 months, rearfoot deformity, ankle osteoarthritis, and posterior tibial tendon dysfunction, ankle dorsiflexion of $<10^{\circ}$, neurological disorders, or rheumatoid arthritis. It is known that Brostrom-Gould modified surgery is an effective approach that strengthens the original ligaments with the extensor retinaculum without sacrificing

Table I. Descriptive analyses of patients in the study.

\begin{tabular}{lrcl}
\hline Variables & \multicolumn{1}{l}{ CAI } & Normal group & p value \\
\hline Age $($ mean $\pm \mathrm{SD})$ & $29.5 \pm 2.7$ & $30.9 \pm 3.2$ & 0.269 \\
\hline Height $(\mathrm{cm})$ & $175.2 \pm 6,0$ & $177.2 \pm 5.25$ & 0.075 \\
\hline Weight,$(\mathrm{kg})$ & $82.6 \pm 9.8$ & $78 \pm 11.2$ & 0.012 \\
\hline D
\end{tabular}

Data are presented with Mean \pm SD (range).

Table II. Study group intrinsic osseous ankle morphological characteristic comparisons.

\begin{tabular}{|c|c|c|c|c|}
\hline & Abbreviation & $\begin{array}{l}\text { Recurrent Inversion } \\
\text { Ankle Sprain Group } \\
(\mathrm{n}=52)\end{array}$ & $\begin{array}{l}\text { Healthy } \\
\text { Control Group } \\
(n=46)\end{array}$ & p-value \\
\hline Tibial Arc Length & TiAL & $29.0 \pm 3.6 \mathrm{~mm}$ & $31.4 \pm 3.6 \mathrm{~mm}$ & 0.001 \\
\hline Sagittal Radius of the Trochlea & SRTi & $23.3 \pm 2.3 \mathrm{~mm}$ & $16.1 \pm 5.4 \mathrm{~mm}$ & $<0.0001$ \\
\hline Trochlea Tali Length & TaAL & $41.3 \pm 4.4 \mathrm{~mm}$ & $29.0 \pm 5.2 \mathrm{~mm}$ & $<0.0001$ \\
\hline Sagittal Radius of the Trochlea Tali Arc & SRTa & $25.9 \pm 2.8 \mathrm{~mm}$ & $25.4 \pm 5.9 \mathrm{~mm}$ & $<0.0001$ \\
\hline Height of Talus & $\mathrm{H}$ & $24.5 \pm 6.2 \mathrm{~mm}$ & $21.2 \pm 4.1 \mathrm{~mm}$ & 0.002 \\
\hline Tibiotalar Sector Angle & $\mathrm{a}$ & $76.8 \pm 6.0^{\circ}$ & $81.0 \pm 6.6^{\circ}$ & 0.001 \\
\hline Ankle Stability Angle & $\gamma$ & $14.0 \pm 2.5^{\circ}$ & $22.1 \pm 7.3^{\circ}$ & $<0.0001$ \\
\hline $\begin{array}{l}\text { Sagittal Radius of the Trochlea: } \\
\text { Sagittal Radius of the Trochlea Tali Arc Ratio }\end{array}$ & SRTi : SRTa & $0.90 \pm 0.07$ & $0.47 \pm 0.19$ & $<0.0001$ \\
\hline
\end{tabular}

Independent sample t-tests were used to determine mean group differences. 
other normal structures, limiting the inversion to stabilize the subtalar joint, and provides a stable ankle $(7,11,13-15)$. Based on our database, it was observed that the Brostrom-Gould procedure was planned for patients with unstable ankle.

An age- and sex- matched control group consisting of 46 healthy adults ( 26 men and 20 women) with no previous ankle sprain injury history was recruited to create a case-control research study design. Subject demographic information is reported in (table II).

To better enable sagittal plane osseous structure identification, standardized lateral and antero-posterior radiographs were obtained with the ankle in neutral weightbearing alignment. Radiographs were restricted to the distal half of the leg. Measurement calibration was obtained using a pure hydroxyapatite phantom. To control for potential magnification effects all radiographs were performed using consistent fixed tube-detector distance. Digital radiographic images were then reviewed by the senior author and an independent radiologist. Eight measurements were performed (16-19) (Sectra Workstation IDS7, version 21.2.8.6195 software, Linköping, Sweden) as shown in figure $\mathbf{1} \mathbf{a}-\mathbf{d}$ and figure $\mathbf{2} \mathbf{a}-\mathbf{d}$. Measurement reliability revealed intraclass correlation coefficients of $\geq 0.75$ (good to excellent reliability). Additionally, the ratio between SRTa:SRTi and TaAL:TiAL was determined. The case group radiographs used for study measurements were taken prior to ankle surgery. Similar to the human glenohumeral joint circle concept these ratios were evaluated to determine the relationship of talus (ball) and tibial surface (socket) dimensions to patient recurrent lateral ankle sprain history (20).

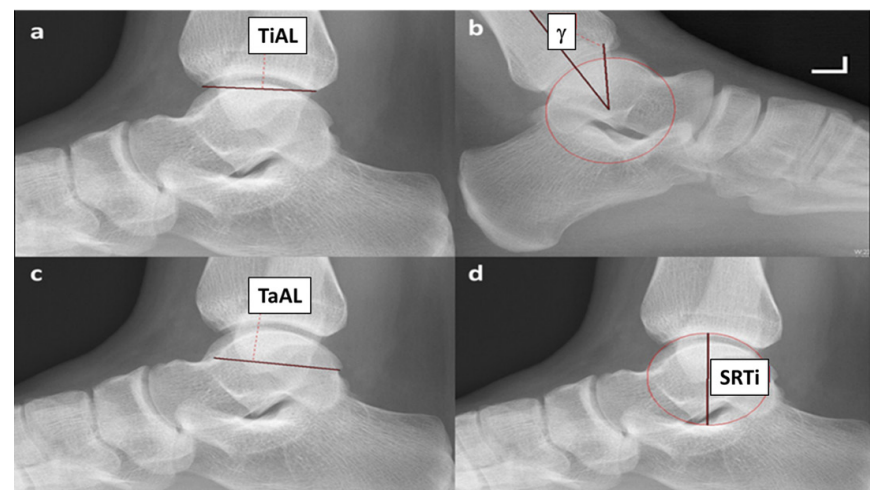

Figure 1. Tibial arc length (TiAL): the sagittal plane length between the most anterior and posterior tibial mortise locations (a); Ankle stability angle ( $\gamma$ ): the angle formed by the tibial axis and a vertical line from the talar dome center with the ankle in maximal plantar flexion (b); Trochlea tali length (TaAL): the length of the line connecting the most anterior and posterior points of the trochlea tali sagittal arc (c); and the Sagittal plane radius of the tibial mortise (SRTi): the radius of the tibial mortise circumference using a least-square fitting technique (d).

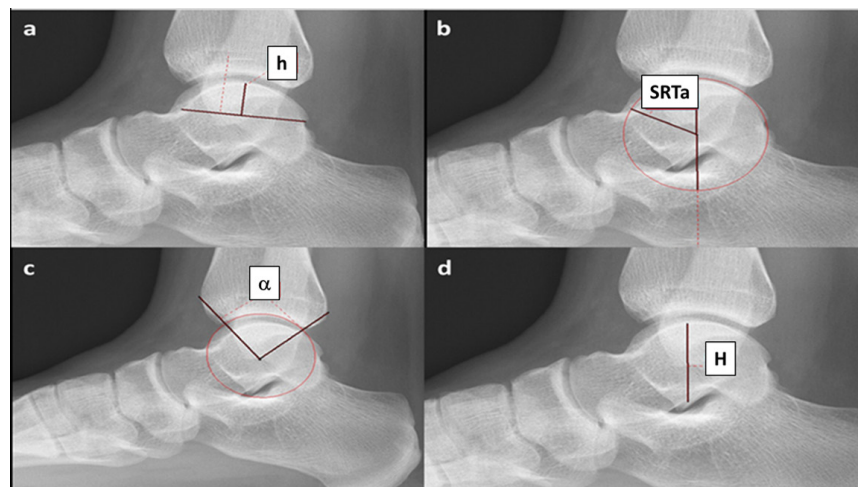

Figure 2. Talar tenon height (h): distance between the talar trochlear dome and the trochlear arch (a); Sagittal plane radius of the trochlea tali arc (SRTa): a circle digitally fit to the talar joint surface approximating the center of the talus. The distance between the talar center and the circle is the radius (b); Tibiotalar sector angle $(\alpha)$ : line from the talar center to the anterior and posterior margins of the distal tibia. These lines enclose the angle a (tibiotalar sector) which defines the size of the tibial coverage of the talus (c); talus height $(\mathrm{H})$ : a line perpendicular to the ground through the talar center representing the distance between the talar surface and the inferior border of the talus (d).

\section{Statistical methods}

Data normality was confirmed using Kolmogorov-Smirnov and Shapiro Wilk tests. Stepwise multiple regression analysis was performed to identify variables that displayed predictive value based on patient recurrent lateral ankle sprain injury history. Following this, independent sample $\mathrm{t}$-tests were used to determine mean group differences. Lastly, receiver operator characteristic (ROC) and area under the curve (AOC) analysis was performed to identify threshold cut-off values for diagnostic test sensitivity and specificity. Post-hoc statistical power analysis was performed using Decision Support Systems Software, SPH Analytics, Alpharetta, GA, USA). All other statistical analysis was performed using SPSS version 26.0 software (IBM-SPSS, Armonk, NY, USA). An alpha value of $\mathrm{p}<0.05$ indicated statistical significance.

\section{RESULTS}

Radiographic intrinsic osseous ankle morphological characteristic values are reported in table II (mean \pm standard deviation). The patient group displayed a shorter TiAL and $\mathrm{h}$ than the healthy control group. The patient group also displayed a greater SRTi, TaAL, SRTa, and $\mathrm{H}$ than the healthy control group. The patient group also displayed lesser $\alpha, \gamma$, SRTi:SRTa ratio, and TiAL:TaAL ratio than the healthy control group. 
Forward stepwise regression with recurrent lateral ankle sprain patient group assignment serving as the dependent variable identified the following independent variables as contributing to a highly predictive statistical model: Patient Group (recurrent lateral ankle sprain history) $=1.45+0.37$ TaAL +-0.43 SRTa $+-0.23 \gamma+0.15 \mathrm{H}+-0.14 \mathrm{~h}+0.17$ SRTi $(\mathrm{P}<0.0001)$. This equation displayed high predictive value with $R=0.96, R^{2}=0.91$. This verified that as TaAL, $H$ and SRTi beta values increased there was a lower likelihood for patient group assignment. As SRTa, $\gamma$, and h beta values decreased there was a greater likelihood for patient group assignment. The variables TiAL and $\alpha$ did not provide statistically significant contributions to the prediction equation. Following this, ROC and AUC analysis of each independent variable was performed to identify which measurements had the greatest sensitivity and specificity for identifying patients with a history of recurrent lateral ankle sprain injury. This revealed that the strongest independent radiographic predictors for the patient group were TaAL (Area = 0.97) and SRTi (Area $=0.84)$ (figure 3). As individual measurements these values displayed generally excellent and good diagnostic value, respectively.

To better delineate their clinical decision-making efficacy, the following ratio values were assessed: SRTi:SRTa and TaAL:TiAL (figure 4). The AOC for the SRTi:SRTa ratio was 0.95 (95\% confidence interval of $0.93-1.0)$. For the SRTi:SRTa ratio, a value of 0.68 produced a sensitivity of 0.96 and a specificity of 0.85 . The AOC for the TaAL:TiAL ratio was 0.97 (95\% confidence interval of $0.89-1.0)$. For the TaAL:TiAL ratio, a value of 1.15 produced a sensitivity of 0.98 and a specificity of 0.91 . Post-hoc statistical power analysis for both ratio values revealed excellent statistical power $(100 \%)$ at an alpha level of 0.05 .

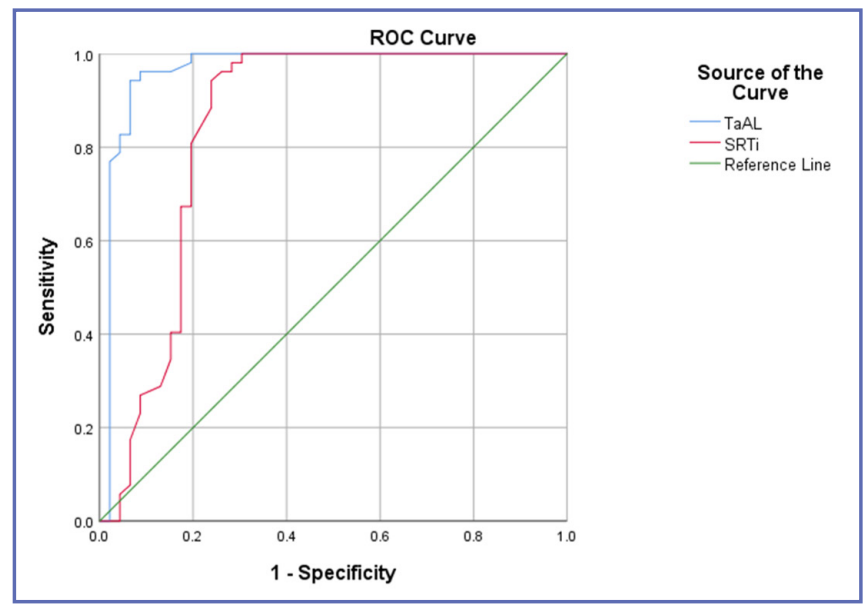

Figure 3. Receiver operating characteristic plots for independent TaAL and SRTi osseous morphology characteristics.

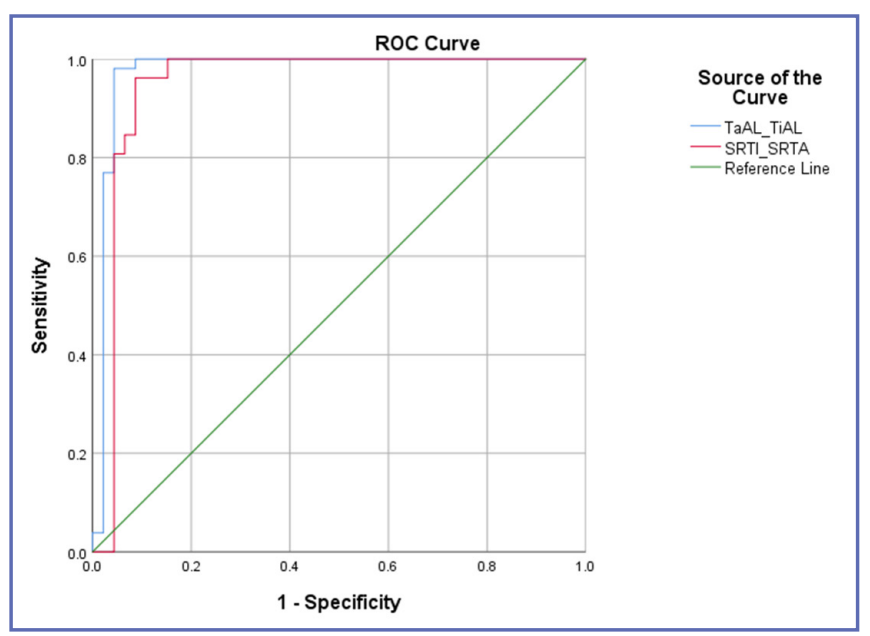

Figure 4. Receiver operating curve characteristics for SRTi: SRTa and TaAL:TiAL osseous characteristic ratios.

\section{DISCUSSION}

The most important result of this study was that recurrent lateral ankle joint stability was directly influenced by intrinsic osseous ankle morphological characteristics. Of greatest clinical significance were the ratio between the sagittal plane radius of the tibial mortis (SRTi) and the sagittal radius of the trochlea tali arc (SRTa) and the ratio between trochlea tali length (TaAL) and tibial arc length (TiAL).

The SRTi:SRTa ratio displayed excellent sensitivity and good specificity. Using this measurement method $95 \%$ of subjects would be classified correctly and only $15 \%$ who were deemed negative would be incorrectly classified. For the TaAL:TiAL ratio, at a value of 1.15 , sensitivity was 0.98 and specificity was 0.91 . This suggests that in using this method, $98 \%$ of subjects would be classified correctly and only $9 \%$ who were deemed negative would be incorrectly classified. In managing patients with recurrent lateral ankle sprain history, these ratio measurements can assist the physician and surgeon who treats a patient with recurrent lateral ankle sprain history as they consider whether or not surgical ankle ligament stabilization may be indicated to achieve a positive outcome.

The ratio measurements we recommend provided excellent sensitivity and very good specificity for distinguishing individuals with the intrinsic osseous ankle morphological characteristics that are consistent with recurrent lateral ankle sprain injury. Individuals with a larger radius, corresponding to a flatter talus, and a smaller tibiotalar sector, corresponding to less talus restraint in the tibia were more likely to have recurrent lateral ankle sprain injury history. In conjunction with a comprehensive clinical examination including patient history, neuromuscular control and capsu- 
loligamentous laxity assessments, radiographic evaluation of these intrinsic risk factors might help doctors and surgeons more effectively make decisions about the potential efficacy of surgical or conservative rehabilitation treatments for improving patient outcomes.

Persistent pain, recurrent sprains, and repeated "giving way" or "buckling" are hallmarks of debilitating chronic ankle instability (21). Although deformity (e.g., rearfoot varus, first ray plantar flexion, mid-foot cavus, and generalized laxity) contributes to ankle sprain predisposition, the combination of mechanical (pathologic laxity, synovial changes, abnormal morphology and degenerative changes) and functional (impaired proprioception, postural control, and neuromuscular control) insufficiencies in association with recurrent lateral ankle sprain injuries are key contributors to chronic ankle instability $(22,23)$.

Osteophyte excision is commonly recommended for patients with anterior ankle impingement (24). In the studies, it has been reported at a rate of $12-26 \%$ and may contribute to decreased tibial mortise coverage of the talus, further disposing it to anterior dislocation and greater ankle $(25,26)$. In these patients, osteophyte excision with ligament repair produces satisfactory results by increasing functional results and range of motion (27). When ankle instability is not diagnosed and treated in a timely manner, $78 \%$ of individuals develop posttraumatic ankle osteoarthritis, being a negative prognostic factor for poor results in terms of functional scores and negatively affecting patients' motivation, and this entails significant healthcare costs (28, 29). The accumulative effects of chronic ankle instability on long-term joint health and disability are not as well understood (30). Whether they originate from an occupational or athletic initial injury mechanism chronic ankle instability or incongruency increases contact stresses and shear forces contribute to articular cartilage degeneration or defect development $(31,32)$.

This study has several limitations. Most importantly, it solely evaluated intrinsic osseous ankle morphological characteristics, not capsuloligamentous or neuromuscular contributions to joint stability. Although the imaging used relied solely on standard clinical uniplanar radiographs, the osse-

\section{REFERENCES}

1. Acevedo JI, Mangone P. Ankle instability and arthroscopic lateral ligament repair. Foot Ankle Clin 2015;20(1):59-69.

2. Buchhorn T, Sabeti-Aschraf M, Dlaska CE, Wenzel F, Graf A, Ziai P. Combined medial and lateral anatomic ligament reconstruction for chronic rotational instability of the ankle. Foot Ankle Int 2011;32(12):1122-6. ous landmark ratios we describe provided insight as to the complex three-dimensional nature of the functional ankle joint (17). Ankle capsuloligamentous and neuromuscular structures provide essential non-contractile and contractile constraints to dynamic ankle joint stability. However, standardized single plane radiographs as commonly ordered during an initial clinical examination potentially possess greater diagnostic value than currently believed. When the ankle is evaluated in terms of chronic instability, the most important intrinsic mechanical factor such as sagittal alignment (SRTi:SRTa and TaAL:TiAL ratio), is often overlooked because functional factors are more prominent. All factors should be considered in a holistic, rehabilitative treatment to better target this condition and reduce health and economic burden. Recently, there is an increasing trend towards earlier surgical treatment in patients with mechanical instability, which reduces or prevents the progress of more serious problems in the future (33).

\section{CONCLUSIONS}

Intrinsic osseous ankle morphological characteristics were related to recurrent lateral ankle sprain injuries. The ratio between the sagittal plane radius of the tibial mortis (SRTi) and the sagittal radius of the trochlea tali arc (SRTa) and the ratio between trochlea tali length (TaAL) and tibial arc length (TiAL) provide excellent sensitivity and good specificity clinical decision-making measurement characteristics. Intrinsic osseous ankle morphological characteristics such as these can help doctors and surgeons make treatment decisions for patients with recurrent lateral ankle sprain injuries.

\section{ACKNOWLEDGMENTS}

We express our gratitude to Prof. E. Turhan, for his assistance.

\section{CONFLICT OF INTERESTS}

The authors declare that they have no conflict of interests.
3. Bozkurt M, Doral MN. Anatomic factors and biomechanics in ankle instability. Foot Ankle Clin 2006;11(3):451-63.

4. Erkman MJ, Walker PS. A study of knee geometry applied to the design of condylar prostheses. Biomed Eng 1974;9(1):14-7.

5. Halabchi F, Angoorani H, Mirshahi M, Pourgharib Shahi MH, Mansournia MA. The Prevalence of Selected Intrinsic Risk 
Factors for Ankle Sprain Among Elite Football and Basketball Players. Asian J Sports Med 2016;7(3):e35287.

6. Kuo CC, Lu HL, Lu TW, et al. Effects of positioning on radiographic measurements of ankle morphology: a computerized tomography-based simulation study. Biomed Eng Online 2013;12:131.

7. Doherty C, Bleakley C, Hertel J, Caulfield B, Ryan J, Delahunt E. Recovery From a First-Time Lateral Ankle Sprain and the Predictors of Chronic Ankle Instability: A Prospective Cohort Analysis. Am J Sports Med 2016;44(4):995-1003.

8. McKeon PO, Mattacola CG. Interventions for the prevention of first time and recurrent ankle sprains. Clin Sports Med 2008;27(3):371-82, viii.

9. van Rijn RM, van Ochten J, Luijsterburg PA, van Middelkoop M, Koes BW, Bierma-Zeinstra SM. Effectiveness of additional supervised exercises compared with conventional treatment alone in patients with acute lateral ankle sprains: systematic review. BMJ 2010;341:c5688.

10. Gribble PA, Delahunt E, Bleakley C, et al. Selection criteria for patients with chronic ankle instability in controlled research: a position statement of the International Ankle Consortium. Br J Sports Med 2014;48(13):1014-8.

11. Gould N, Seligson D, Gassman J. Early and late repair of lateral ligament of the ankle. Foot Ankle 1980;1(2):84-9.

12. Pourkazemi F, Hiller CE, Raymond J, Black D, Nightingale EJ, Refshauge KM. Predictors of recurrent sprains after an index lateral ankle sprain: a longitudinal study. Physiotherapy 2018;104(4):430-7.

13. Cao Y, Hong Y, Xu Y, Zhu Y, Xu X. Surgical management of chronic lateral ankle instability: a meta-analysis. J Orthop Surg Res 2018;13(1):159.

14. Gerstner Garces JB. Chronic ankle instability. Foot Ankle Clin 2012;17(3):389-98.

15. Maffulli N, Del Buono A, Maffulli GD, et al. Isolated anterior talofibular ligament Broström repair for chronic lateral ankle instability: 9-year follow-up. Am J Sports Med 2013;41(4):858-64.

16. Kwon DG, Sung KH, Chung CY, et al. Preliminary findings of morphometric analysis of ankle joint in Korean population. J Foot Ankle Surg 2014;53(1):3-7.

17. Leardini A. Geometry and mechanics of the human ankle complex and ankle prosthesis design. Clin Biomech (Bristol, Avon) 2001;16(8):706-9.

18. Leardini A, Belvedere C, Nardini F, Sancisi N, Conconi M, Parenti-Castelli V. Kinematic models of lower limb joints for musculo-skeletal modelling and optimization in gait analysis. J Biomech 2017;62:77-86.

19. Stagni R, Leardini A, Catani F, Cappello A. A new semi-automated measurement technique based on X-ray pictures for ankle morphometry. J Biomech 2004;37(7):1113-8.

20. Warren RF, Kornblatt IB, Marchand R. Static factors affecting posterior shoulder stability. Orthop Trans 1984;8:89.

21. Evans DL. Recurrent instability of the ankle; a method of surgical treatment. Proc R Soc Med 1953;46(5):343-4.

22. Frigg A, Magerkurth O, Valderrabano V, Ledermann HP, Hintermann B. The effect of osseous ankle configuration on chronic ankle instability. Br J Sports Med 2007;41(7):420-4.

23. Ferran NA, Oliva F, Maffulli N. Ankle instability. Sports Med Arthrosc Rev 2009;17(2):139-45.

24. Ross KA, Murawski CD, Smyth NA, et al. Current concepts review: Arthroscopic treatment of anterior ankle impingement. Foot Ankle Surg 2017;23(1):1-8.

25. Odak S, Ahluwalia R, Shivarathre DG, et al. Arthroscopic Evaluation of Impingement and Osteochondral Lesions in Chronic Lateral Ankle Instability. Foot Ankle Int 2015;36(9):1045-9.

26. Hua Y, Chen S, Li Y, Chen J, Li H. Combination of modified Broström procedure with ankle arthroscopy for chronic ankle instability accompanied by intra-articular symptoms. Arthroscopy 2010;26(4):524-8.

27. Yang Q, Zhou Y, Xu Y. Arthroscopic debridement of anterior ankle impingement in patients with chronic lateral ankle instability. BMC Musculoskelet Disord 2018;19(1):239.

28. Harrington KD. Degenerative arthritis of the ankle secondary to long-standing lateral ligament instability. J Bone Joint Surg Am 1979;61(3):354-61.

29. Shah S, Thomas AC, Noone JM, Blanchette CM, Wikstrom EA. Incidence and Cost of Ankle Sprains in United States Emergency Departments. Sports Health 2016;8(6):547-52.

30. Bosien WR, Staples OS, Russell SW. Residual disability following acute ankle sprains. J Bone Joint Surg Am 1955;37-a(6):1237-43.

31. Hintermann B. Medial ankle instability. Foot Ankle Clin 2003;8(4):723-38.

32. van Ochten JM, de Vries AD, van Putte N, et al. Association between Patient History and Physical Examination and Osteoarthritis after Ankle Sprain. Int J Sports Med 2017;38(9):717-24.

33. Michels F, Pereira H, Calder J, et al. Searching for consensus in the approach to patients with chronic lateral ankle instability: ask the expert. Knee Surg Sports Traumatol Arthrosc 2018;26(7):2095-102. 